\title{
Classification of Breast Cancer Cell Images using Multiple Convolution Neural Network Architectures
}

\author{
Zarrin Tasnim ${ }^{1}$, F. M. Javed Mehedi Shamrat ${ }^{2}$, Md Saidul Islam ${ }^{3}$ \\ Md.Tareq Rahman ${ }^{4}$, Biraj Saha Aronya ${ }^{5}$, Jannatun Naeem Muna ${ }^{6}$, Md. Masum Billah ${ }^{7}$ \\ Department of Software Engineering, Daffodil International University, Bangladesh ${ }^{1,2,5,7}$ \\ School of Computer and Software, Nanjing University of Information Science and Technology, China ${ }^{3}$ \\ Department of Computer Science and Engineering, Daffodil International University, Bangladesh ${ }^{4}$ \\ Department of Computer Science and Engineering, United International University, Bangladesh ${ }^{6}$
}

\begin{abstract}
Breast cancer is a malignant tumor that affects women. It is the most prevalent cancer in women, affecting about $10 \%$ of all women at any point in their lives. The development of breast cancer begins in the lobules or ducts of the cells. Early detection and prevention are the best ways to stop this cancer from spreading. In this study, five Convolution Neural Network (CNN) models are used to process image data of breast cells. AlexNet, InceptionV3, GoogLeNet, VGG19 and Xception models are used for the classification of Invasive Ductal Carcinoma, IDC and Non-Invasive Ductal Carcinoma (Non-IDC) cells. The models are trained and tested at different epochs to record the learning rate. It is observed from the study that with higher epochs, the data loss decreases and accuracy increases. The accuracy of InceptionV3 and Xception is $92.48 \%$ and $90.72 \%$ respectively. Likewise, VGG19 and AlexNet have fairly close accuracy of $94.83 \%$ and $96.74 \%$. However, GoogLeNet dominates over the other implemented models with the highest accuracy of $97.80 \%$. The GoogLeNet model performs with high accuracy and precision in detecting IDC cells responsible for breast cancer.
\end{abstract}

Keywords-Breast cancer; IDC; non-IDC; AlexNet; VGG19; Inception sV3; GoogLeNet; Xecption; accuracy

\section{INTRODUCTION}

Cancer, also known as a malignant neoplasm, is a group of more than a hundred diseases marked by irregular cell development with the ability to spread to the body's underlying tissues. IDC is a kind of breast cancer that started in the ducts of the breast and has progressed to fibrous or fatty tissue outside of the duct. IDC is the most prevalent kind of breast cancer, accounting for $80 \%$ of all occurrences. Breast cancer is the most common kind of cancer in women worldwide [1]. Many imaging techniques have been developed to aid in the early diagnosis and treatment of breast cancer, as well as the reduction of breast cancer-related mortality. To improve diagnostic precision and accuracy, many assisted breast cancer diagnosis methods have been employed [2-4]. Fig. 1 shows breast cancer cases around the world.

To classify and predict breast cancer, machine learning algorithms with image processing have become quite famous for their accuracy in detecting the disease at an early stage. Ciresan et al. [5] classified each pixel into mitotic and nonmitotic groups using an 11-layered CNN. The predictions were made using likelihood ratings allocated to each pixel depending on its distance from the mitosis centroid. A related study [6] used Transfer Learning in CNNs to identify and segment brain and colon cancer images, and the findings were cutting-edge. It used AlexNet (pre-trained on ImageNet) to train a Support Vector Machine with the features extracted from the last FC layer Support Vector Machine (SVM). Gao et al. used CNN to identify interstitial lung infections [7] and discovered that a pre-trained model converged categorization faster than a randomly initialized network. It is possible to automate cell counting in microscope pictures. Weidi et al. [8] took a regression approach to the issue, which eliminates the need for previous identification or segmentation. They regressed a density surface generated by the superposition of Gaussians using completely convolutional regression networks (FCRNs). The dot annotations of each cell given as the ground truth for the training set are expressed by these Gaussians. To identify the best-supervised learning classifier, Vikas Chaurasia and Saurabh Pal [9] evaluate the performance criteria of Naive Bayes, SVM-RBF kernel, RBF neural networks, Decision trees, and basic CART in breast cancer datasets. The experimental results indicate that the SVM-RBF kernel outperforms other classifiers, scoring 96.84\% accuracy in the Wisconsin Breast Cancer (original) datasets. Djebbari et al. [10] investigate the impact of an ensemble of machine learning approaches on breast cancer survival period prediction. When compared to prior results, their methodology is more accurate on their breast cancer data collection. S. Aruna and L. V Nandakishore [11] compare the findings of C4.5, Nave Bayes, Support Vector Machine (SVM), and KNearest Neighbor to find the appropriate classifier in WBC (K$\mathrm{NN})$. SVM is the most accurate classifier, with a $96.99 \%$ accuracy rate. Angeline Christobel. Y. [12] use a decision tree classifier (CART) to obtain an accuracy of $69.23 \%$ in breast cancer datasets. The accuracy of data mining algorithms SVM, IBK, and BF Tree is compared by A. Pradesh [13]. SMO outperforms other classifiers in terms of performance. T.Joachims [14] uses neuron fuzzy methods to reach a precision of $95.06 \%$ by utilizing Wisconsin Breast Cancer (original) datasets. In this study, a hybrid method is proposed to increase the classification accuracy of Wisconsin Breast Cancer (original) datasets using 10-fold cross-validation. Liu Ya-Qin, W. Cheng, and Z. Lu [15] used the C5 algorithm with picking to produce additional data for training from the initial array using variations of repetitions to yield multisets of the same scale as the original data to predict breast cancer survivability. Delen et al. Lu [16] pre-classified 202,932 breast 
cancer medical records into two groups: those who "survived" $(93,273)$ and those who "didn't" $(93,272)$. (109,659). The precision of the prediction of survivability was in the region of $93 \%$.

Estimated number of new cases in 2020 , worldwide, females, all ages

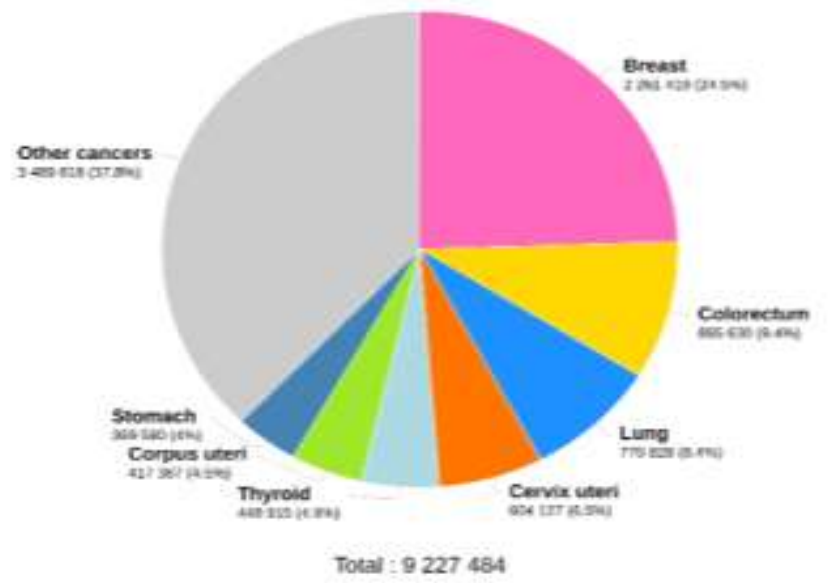

Fig. 1. The Distribution of the Confirmed Breast Cancer Cases around the World (2020). (Source: World Health Organization).

In this work, to detect cancer cells in time for fast treatment, CNN models are used to classify the IDC cells in the breast to determine breast cancer. The CNN models proposed for the study are AlexNet, GoogLeNet, VGG19, Xception and InceptionV3. The aim is to classify IDC from Non-IDC breast cell images from the dataset. Furthermore, the accuracy of the models is compared with each other to determine which model performs best.

The remainder of this paper is organized as follows. Section II contains the overall view of the system. It gives an idea of how the study is conducted in each step. Section III comprises the materials and the methods in detail. It also explains the criteria under which the performance of the implemented models will be evaluated. Section IV summarizes the experimental studies and the obtained results. Section V provides a comparison of the proposed system with the existing studies to show that the proposed system has superior performance over others. Finally, Section VI presents the conclusion of the study.

\section{PROPOSED SYSTEM}

Early detection of breast cancer is a critical field on which researchers are working since it may improve the rate of diagnosis, care, and recovery of affected women. Early identification is the most important measure in reducing this condition's clinical and social risks, given the high expense of care and the high incidence of the disease among women worldwide. There are several approaches and techniques for detecting this form of cancer, each with its own set of benefits and drawbacks. When cancer has spread through the later phases, it is usually identified and diagnosed. This is especially bad since cancer risks have metastasized by the time it is discovered are large, leaving the chances of treating it very low. Self-testing is rarely done, which tends to cancer detection in its latter stages. A lump or mass on the breast, selfexamination, or mammography is the most common way to diagnose breast cancer.

In the proposed system, image data of breast cells are used to predict breast cancer. For that, the images are classified to identify which cells cause cancer. The models used for the prediction are AlexNet, IceptionV3, VGG19, Xception and GoogLeNet. An overall flow diagram of the study is presented in Fig. 2.

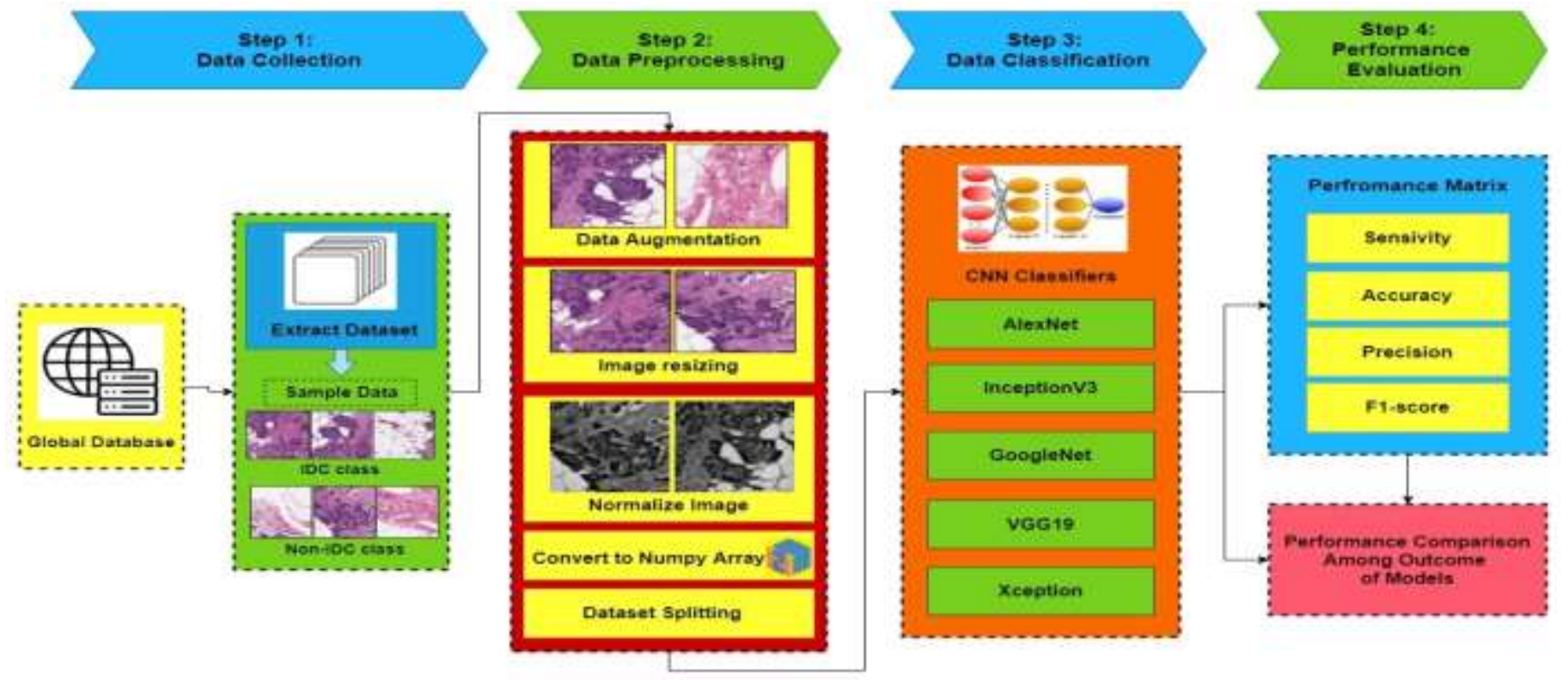

Fig. 2. System Flow Diagram of the Proposed Study. 


\section{SYSTEM OPERATION}

\section{A. Data Description}

The image used in this study is of breast cells to diagnose Breast cancer. The dataset was retrieved from "https://www. kaggle.com/paultimothymooney/breast-histopathology-images/ discussion/130203". For training and testing the machine learning models, 27800 image data are used. The images are categorized into two categories,

- Non-IDC: categorized as "class0".

- IDC: categorized as "class1".

It will help specify IDC (Malignant (cells are abnormal and grow uncontrollably)) and Non-IDC (Benign (if the cells are normal just overgrown)).

Several IDC and non-IDC image data were added for better training of the models to enrich the dataset. Fig. 3 and Fig. 4 contain some of the IDC and non-IDC class images that were added to the dataset, respectively.

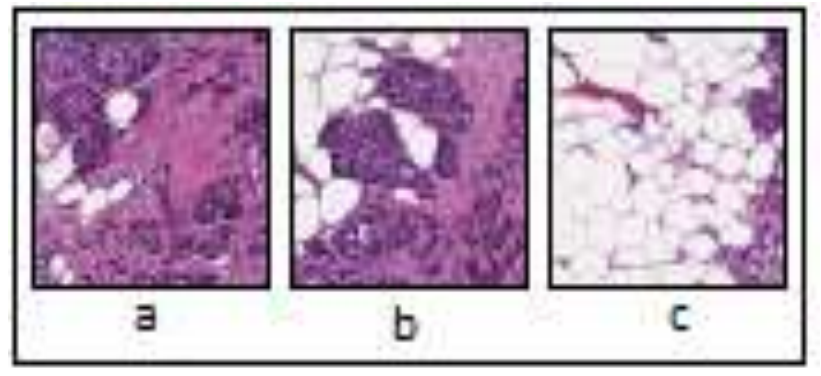

Fig. 3. Image Data of IDC Class.

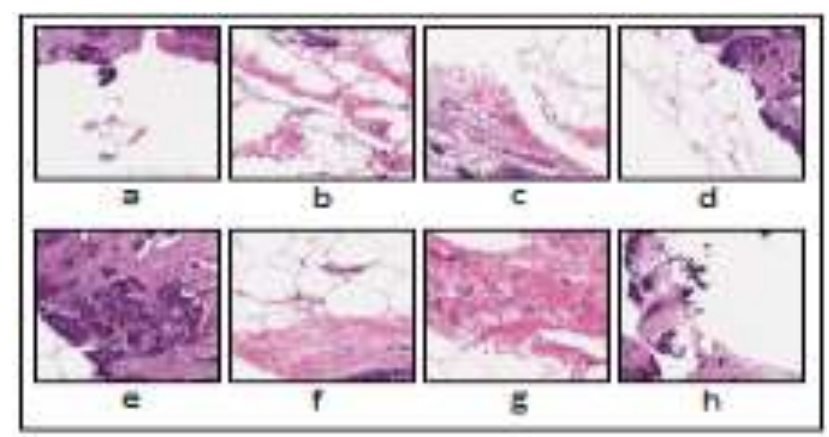

Fig. 4. Image Data of non-IDC Class.

\section{B. Data Pre-processing}

The dataset contains raw image data that are not fit for models to be trained and tested. To bring all the raw data into the same scale, data pre-processing is done on the dataset used in the work. The steps of the pre-processing stage are as follows.

- Generating augmented image: to train any machine learning algorithms, a large dataset is required. To increase the dataset volume, augmented images are generated using the ImageDataGenerator class in the Keras library. Table I holds the attributes used for image generation.
- Resize image: Since all images in the dataset are not in the same size, all the images are reshaped to $128 \mathrm{X} 128$ pixels.

- Normalize image: since all the images are in RBG, converting to greyscale images is being divided by 255 for normalization.

- Convert to NumPy array: image data a converted to NumPy array for faster computation.

- Dataset Splitting: the dataset is split into 80:20 for training and testing the models.

TABLE I. IMAGEDATAGENERATOR ATTRIBUTES

\begin{tabular}{|l|l|}
\hline Shear Range & 0.3 \\
\hline Zoom_Range & 0.2 \\
\hline Horizontal_Flip & True \\
\hline Vertical_Flip & True \\
\hline Rescale & $1 / 255$ \\
\hline
\end{tabular}

C. CNN Models of Classification

1) AlexNet: AlexNet [17] is an 8-layered network with 5 convolutional layers and 3 Max Pooling layers [18]. ReLU activation is used. 96 filter sizes with a stride of 4 give the first Convolution sheet. After that, the 3X3 inputs go into the Max Pooling Sheet, with a stride of 2 . Then, the data is sent to the second convolution layer with stride one and padding two, with a total of $2565 . x / 2$. The data is then followed by a second datasheet, where the stride is 2 and the filter size is 3 . Three Convolution layers with 384, 384, 384, and 256 kernels are then applied to the input results, followed by an Activation layer with 3 X 3 kernels, followed by a Reshape layer with 512 kernels and a padding value of 1 . With pool size 3 , the final MaxPooling is implemented. If all the operations have been performed, the results are transferred to three connected layers, which are eventually converted into totally connected layers. In Fig. 5, the architecture is seen.

2) InceptionV3: InceptionV3 [19] is the third iteration of Google's Inception Convolutional Neural Network, which was first shown at the ImageNet Recognition Challenge. It includes Label Smoothing, Factorized 7X7 Convolution, RMSProp Optimizer, BatchNorm in the Auxillary Classifiers and a downscaling classifier to identify and add information from smoothed label sequences. This is shown in Fig. 6.

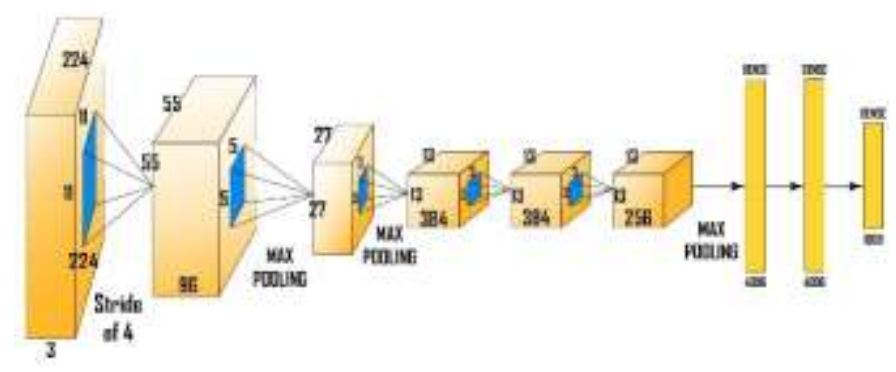

Fig. 5. AlexNet Architecture. 


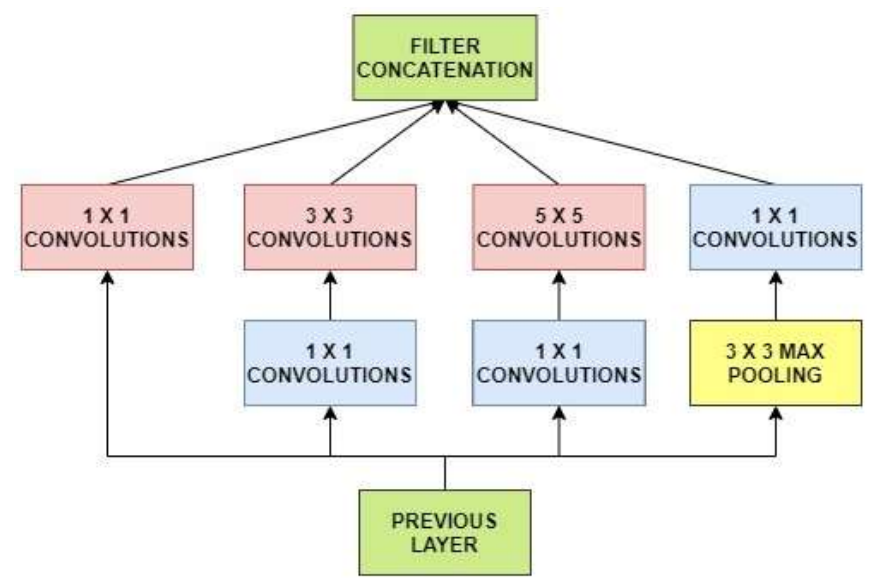

Fig. 6. Implemented InceptionV3 Structure.

3) GoogLeNet: Since 'InceptionV1' [20] is sometimes referred to as GoogLeNet [21]. There are 47 stages of aggregation and several pooling layers in GoogLeNet. A result is that, to sum up, the nine Inception modules are lined up one after another. In the case of GoogLeNet, the stochastic descent algorithm is employed. The following Fig. 7 is an example.

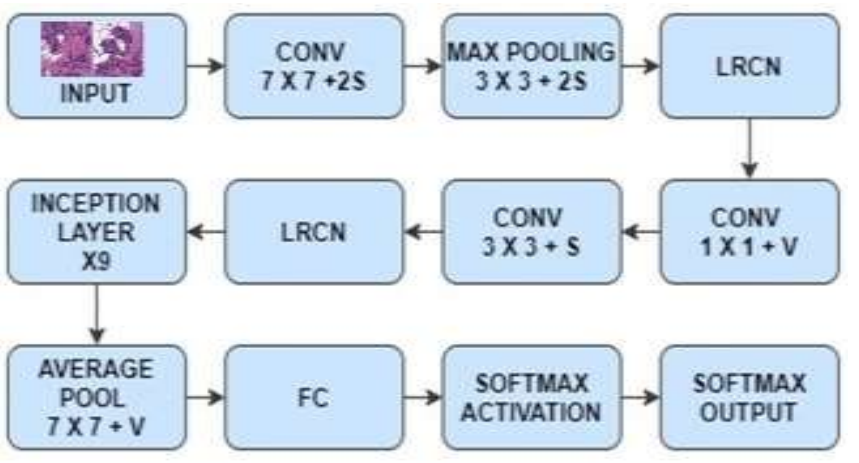

Fig. 7. GoogLeNet Model.

4) VGG19: VGG19 is a version of the VGG model that includes 16 convolutional layers, three fully connected layers, five MaxPool layers, and one SoftMax layer as shown in Fig. 8. A fixed-size RGB picture was used as the input to this network, which also has a matrix of the same size. Max pooling was done using sride 2 across a $2 * 2$ pixel window. This was followed by the Rectified linear unit (ReLu) to add non-linearity into the model in order to enhance classification and computing speed. Three completely linked layers were implemented. And finally, a softmax function is used as the last layer.

5) Xception: The Xception model's base layer is initially frozen with the command (include top=False), followed by the trainable layer as shown in Fig. 9. The trainable layer employs images that have undergone the Average Pooling procedure. The Average Pooling pool size is $(7,7)$, and there are 128 hidden nodes accessible in this layer. The Adam Stochastic gradient descent method is utilized for optimization, and the ReLu activation function is employed in that layer. Following that, in the output layer, the Softmax activation function is utilized to identify IDC cells using two nodes. A learning rate of 0.01 is specified for backpropagation.

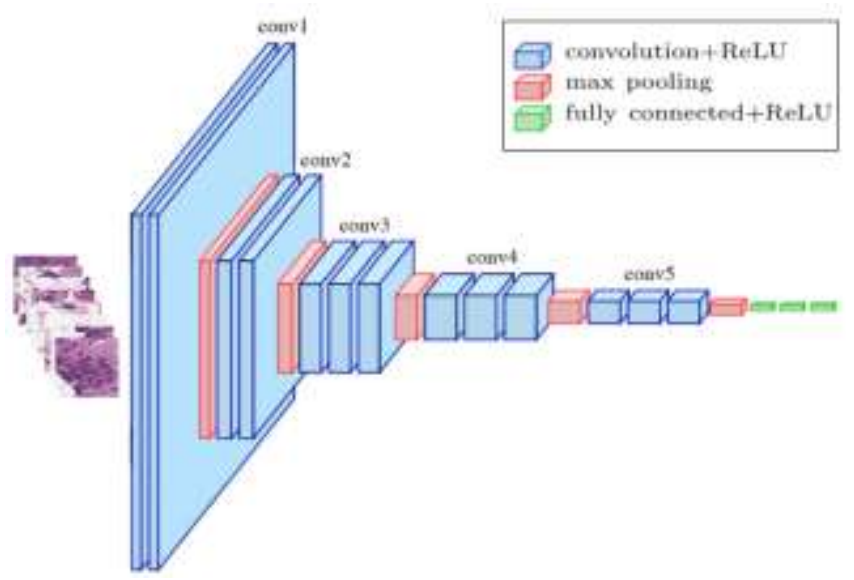

Fig. 8. Applied InceptionV3 Structure.

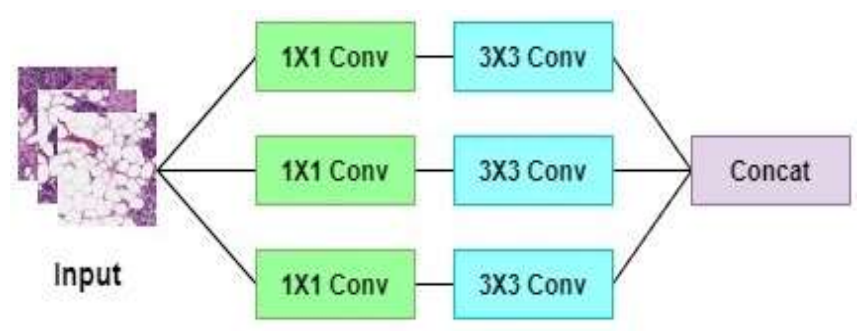

Fig. 9. Implemented Xception Model.

\section{Performance Evaluation}

After completing the training and testing process, the performance of the models is calculated [22, 23]. The evaluation criteria are precision, recall, f1-score, and accuracy, as described in Eq. 1, 2, 3, and 4.

Precision $=\frac{T P}{T P+F P}$
Recall $=\frac{T P}{T P+F N}$
Accuracy $=\frac{T P+T N}{T P+F P+T N+F N}$
$F 1-$ Score $=\frac{\text { Recall } * \text { Precision }}{\text { Recall }+ \text { Precision }}$

\section{RESUlt AND ANALYSIS}

Using the CNN models Vgg19, Xception, AlexNet, InceptionV3, and GoogLeNet, breast cancer prediction can be done successfully. The models can efficiently classify IDC cells and non-IDC cells. However, not all the models perform the same. Some show more accuracy than others.

The outcome was recorded for up to 10 epochs. Table II illustrates the recorded data for the InceptionV3 model where the highest accuracy is $92.48 \%$.

Table III contains the record of accuracy and data loss of the GoogLeNet model for the epoch. It can be observed from the table that with every new epoch, the rate of data loss gradually decreases and the accuracy increase for both the training and test set. 
TABLE II. TRAINING AND TESTING OUTCOME FOR INCEPTION V3

\begin{tabular}{|l|l|l|l|l|}
\hline Epochs & $\begin{array}{l}\text { Training } \\
\text { Data Loss }\end{array}$ & $\begin{array}{l}\text { Training } \\
\text { accuracy in \% }\end{array}$ & $\begin{array}{l}\text { Testing } \\
\text { Data loss }\end{array}$ & $\begin{array}{l}\text { Testing } \\
\text { accuracy in \% }\end{array}$ \\
\hline 1 & 13.29 & 77.34 & 10.21 & 83.42 \\
\hline 2 & 11.02 & 81.93 & 6.46 & 89.94 \\
\hline 3 & 7.76 & 85.67 & 6.39 & 90.24 \\
\hline 4 & 7.39 & 86.76 & 6.23 & 90.73 \\
\hline 5 & 7.56 & 88.34 & 5.93 & 91.23 \\
\hline 6 & 7.26 & 87.99 & 5.74 & 91.47 \\
\hline 7 & 6.98 & 89.23 & 5.46 & 91.78 \\
\hline 8 & 7.23 & 88.80 & 5.28 & 92.12 \\
\hline 9 & 7.34 & 88.23 & 4.97 & 92.48 \\
\hline 10 & 7.03 & 88.57 & 5.01 & 92.10 \\
\hline
\end{tabular}

TABLE III. TRAINING AND TESTING OUTCOME FOR GOOGLENET

\begin{tabular}{|l|l|l|l|l|}
\hline Epochs & $\begin{array}{l}\text { Training } \\
\text { Data Loss }\end{array}$ & $\begin{array}{l}\text { Training } \\
\text { accuracy in \% }\end{array}$ & $\begin{array}{l}\text { Testing } \\
\text { Data loss }\end{array}$ & $\begin{array}{l}\text { Testing } \\
\text { accuracy in \% }\end{array}$ \\
\hline 1 & 9.39 & 93.53 & 7.14 & 94.54 \\
\hline 2 & 5.28 & 95.39 & 4.24 & 96.83 \\
\hline 3 & 5.19 & 95.73 & 4.19 & 97.23 \\
\hline 4 & 5.03 & 95.93 & 3.95 & 97.53 \\
\hline 5 & 4.83 & 96.23 & 3.69 & 97.46 \\
\hline 6 & 4.90 & 96.15 & 3.53 & 97.94 \\
\hline 7 & 4.85 & 96.39 & 3.45 & 97.45 \\
\hline 8 & 4.72 & 96.32 & 3.33 & 97.23 \\
\hline 9 & 4.79 & 96.83 & 3.49 & 97.42 \\
\hline 10 & 4.67 & 96.45 & 3.19 & 97.80 \\
\hline
\end{tabular}

The record of outcome accuracy of AlexNet has stated in Table IV with data loss in every epoch. The highest rate of accuracy rate of AlexNet model is $96.74 \%$ in the test set with a data loss rate of $9.59 \%$ at the $10^{\text {th }}$ epoch. At the same epoch, it achieved the highest accuracy rate on the training set as well with $96.34 \%$.

TABLE IV. TRAINING AND TeSting OUtCome FOR AleXNet

\begin{tabular}{|l|l|l|l|l|}
\hline Epochs & $\begin{array}{l}\text { Training } \\
\text { Data Loss }\end{array}$ & $\begin{array}{l}\text { Training } \\
\text { accuracy in \% }\end{array}$ & $\begin{array}{l}\text { Testing } \\
\text { Data loss }\end{array}$ & $\begin{array}{l}\text { Testing } \\
\text { accuracy in \% }\end{array}$ \\
\hline 1 & 29.26 & 89.93 & 25.77 & 91.01 \\
\hline 2 & 25.18 & 91.37 & 20.54 & 92.80 \\
\hline 3 & 22.06 & 92.32 & 17.28 & 93.59 \\
\hline 4 & 20.62 & 93.34 & 15.66 & 94.11 \\
\hline 5 & 17.97 & 93.73 & 15.23 & 94.21 \\
\hline 6 & 14.49 & 95.06 & 14.98 & 95.87 \\
\hline 7 & 14.44 & 95.45 & 10.10 & 96.23 \\
\hline 8 & 10.29 & 96.11 & 9.89 & 96.19 \\
\hline 9 & 9.87 & 96.72 & 9.48 & 96.33 \\
\hline 10 & 9.93 & 96.34 & 9.59 & 96.74 \\
\hline
\end{tabular}

Table $\mathrm{V}$ contains the record of accuracy and data loss of VGG19 model with respect to epoch. The rate of data loss gradually decreases as the accuracy increase for both the training and test set. The model gives an accuracy of $94.83 \%$ with 5.1 data loss.

Table VI contains the record of outcome accuracy of the Xception model implemented in the dataset, along with data loss for each epoch. Xception model achieves an accuracy rate of $90.72 \%$ in the test set, with a data loss rate of 7.21 at the 8th epoch.

From the recorded data is can be observed that on the 10th epoch, all the models show the highest accuracy and lowest data loss for both train and testing data. A graphical comparison of the accuracy for training (a) and testing (b) data for the models are depicted in Fig. 10 as well.

The data loss rate for the models decreases for both training and test set with every increasing epoch for all the models. The graphs in Fig. 11 show the rate of data loss obtained in each epoch as the model learns from the training (a) and testing (b), the less data it losses.

TABLE V. TRAINING AND TESTING OUTCOME FOR VGG19

\begin{tabular}{|l|l|l|l|l|}
\hline Epochs & $\begin{array}{l}\text { Training } \\
\text { Data Loss }\end{array}$ & $\begin{array}{l}\text { Training } \\
\text { accuracy in \% }\end{array}$ & $\begin{array}{l}\text { Testing } \\
\text { Data loss }\end{array}$ & $\begin{array}{l}\text { Testing } \\
\text { accuracy in \% }\end{array}$ \\
\hline 1 & 13.85 & 84.63 & 9.85 & 90.36 \\
\hline 2 & 11.53 & 87.44 & 9.37 & 91.18 \\
\hline 3 & 10.55 & 89.27 & 8.77 & 91.74 \\
\hline 4 & 9.45 & 89.94 & 7.23 & 92.58 \\
\hline 5 & 9.46 & 89.66 & 7.19 & 92.83 \\
\hline 6 & 8.57 & 90.57 & 7.85 & 92.47 \\
\hline 7 & 9.35 & 90.48 & 5.55 & 94.66 \\
\hline 8 & 8.35 & 91.38 & 5.10 & 94.83 \\
\hline 9 & 8.02 & 91.65 & 6.02 & 94.57 \\
\hline 10 & 8.33 & 91.19 & 6.16 & 94.46 \\
\hline
\end{tabular}

TABLE VI. TRAINING AND TESTING OUTCOME FOR XCEPTION

\begin{tabular}{|l|l|l|l|l|}
\hline Epochs & $\begin{array}{l}\text { Training } \\
\text { Data Loss }\end{array}$ & $\begin{array}{l}\text { Training } \\
\text { accuracy in \% }\end{array}$ & $\begin{array}{l}\text { Testing } \\
\text { Data loss }\end{array}$ & $\begin{array}{l}\text { Testing } \\
\text { accuracy in \% }\end{array}$ \\
\hline 1 & 16.46 & 79.46 & 12.34 & 83.26 \\
\hline 2 & 15.33 & 81.24 & 10.73 & 85.63 \\
\hline 3 & 14.63 & 82.84 & 9.27 & 87.78 \\
\hline 4 & 10.53 & 85.59 & 9.74 & 87.38 \\
\hline 5 & 10.12 & 86.94 & 9.49 & 87.26 \\
\hline 6 & 10.11 & 86.47 & 8.74 & 88.57 \\
\hline 7 & 9.73 & 86.88 & 8.37 & 88.39 \\
\hline 8 & 9.48 & 87.37 & 7.21 & 90.72 \\
\hline 9 & 9.46 & 88.24 & 7.64 & 90.48 \\
\hline 10 & 9.53 & 88.63 & 7.48 & 90.38 \\
\hline
\end{tabular}




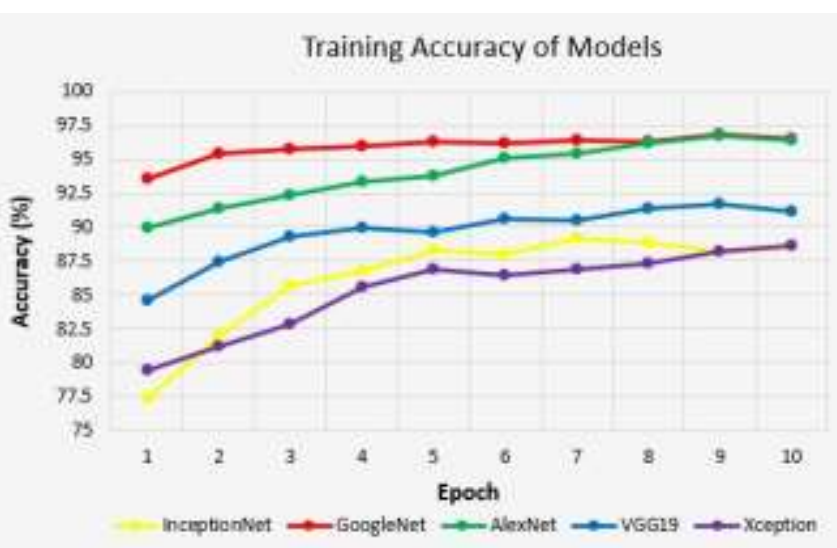

(a)

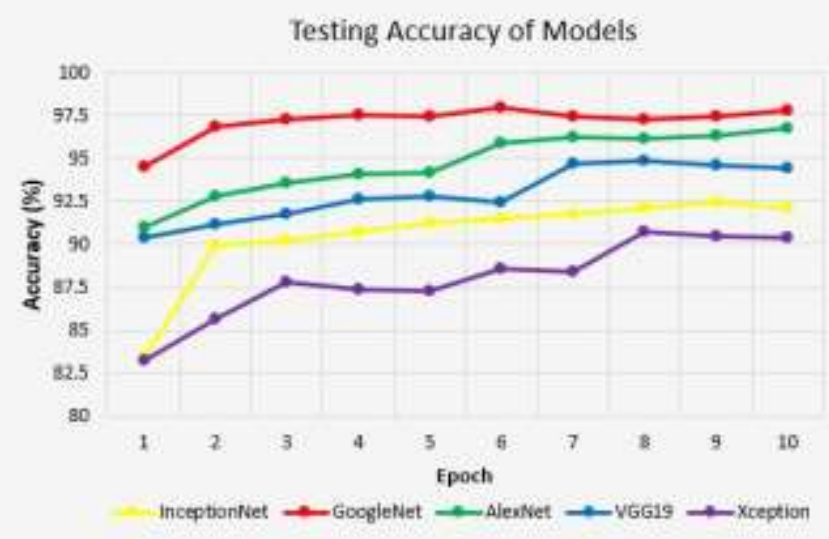

(b)

Fig. 10. Accuracy Comparison for (a) Training Data and (b) Testing Data of the Models.

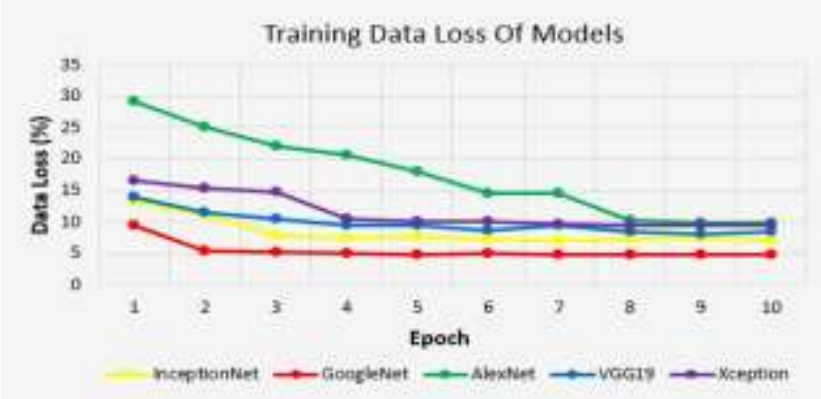

(a)

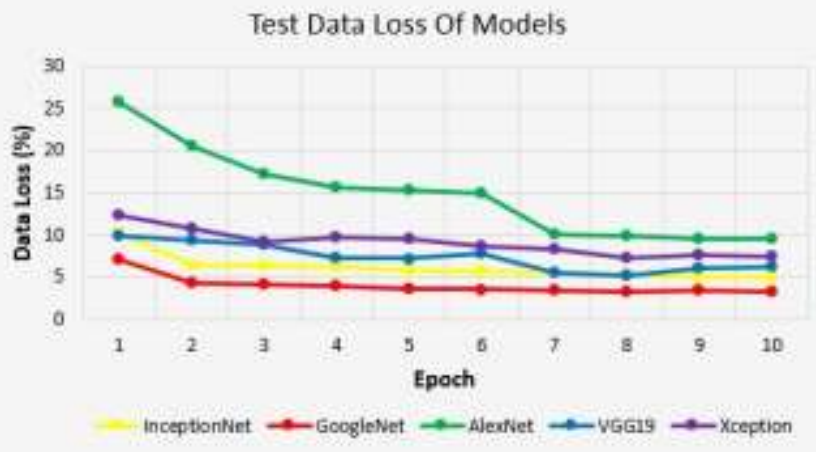

(b)

Fig. 11. Data Loss Comparison for (a) Training Data and (b) Testing Data of the Models.
From the comparison, it is understood that GoogLeNet demonstrates much higher accuracy in predicting breast cancer and classifying cancer cells over other models. For further clarity, and overall performance measurement of the models is calculated in Table VII.

From Fig. 12, it can be deduced that GoogLeNet shows a higher rate for precision, recall and F1-score $(97.34 \%, 96.46 \%$, and $96.46 \%$ ) along with the highest accuracy in predicting breast cancer.

GoogLeNet shows much superior classification performance over InceptionV3 and AlexNet. The models can successfully classify IDC and Non-IDC cells with 97.8\% accuracy. A classification outcome of GoogLeNet for both classes is demonstrated in Fig. 13.

TABLE VII. ClassificAtion REPORT OF TEST DATA

\begin{tabular}{|l|l|l|l|l|}
\hline \multirow{2}{*}{ Models } & \multicolumn{4}{|l|}{ Performance Measures } \\
\cline { 2 - 5 } & Precision & Recall & Accuracy & F1-score \\
\hline Inception v3 & $89.84 \%$ & $90.12 \%$ & $92.48 \%$ & $89.54 \%$ \\
\hline GoogLeNet & $97.34 \%$ & $96.46 \%$ & $97.80 \%$ & $96.46 \%$ \\
\hline Alexnet & $95.12 \%$ & $93.54 \%$ & $96.74 \%$ & $94.65 \%$ \\
\hline VGG19 & $93.63 \%$ & $92.47 \%$ & $94.83 \%$ & $91.36 \%$ \\
\hline Xception & $89.49 \%$ & $91.33 \%$ & $90.72 \%$ & $90.03 \%$ \\
\hline
\end{tabular}

\section{Performance Measurements of the models}

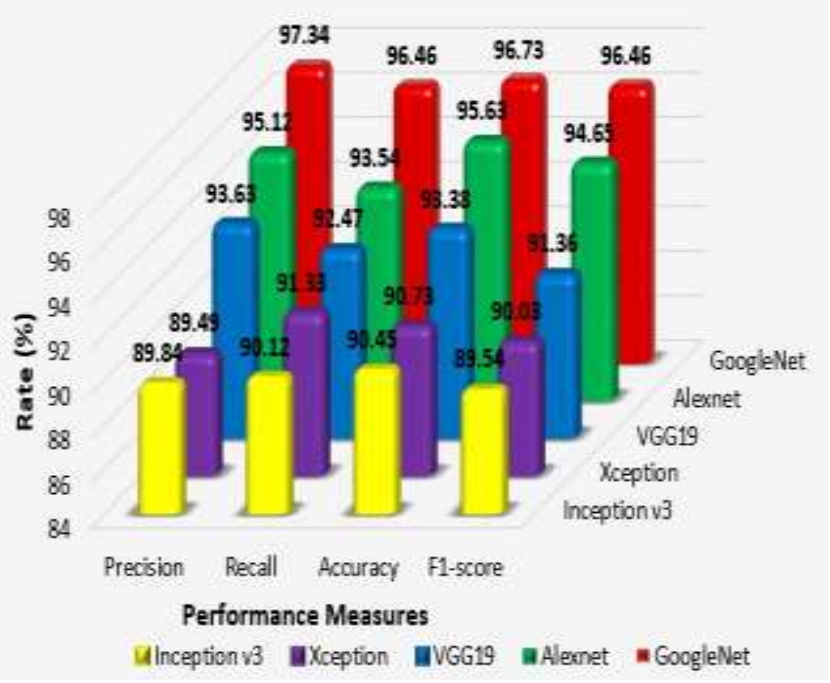

Fig. 12. Performance Evaluation of the Models.

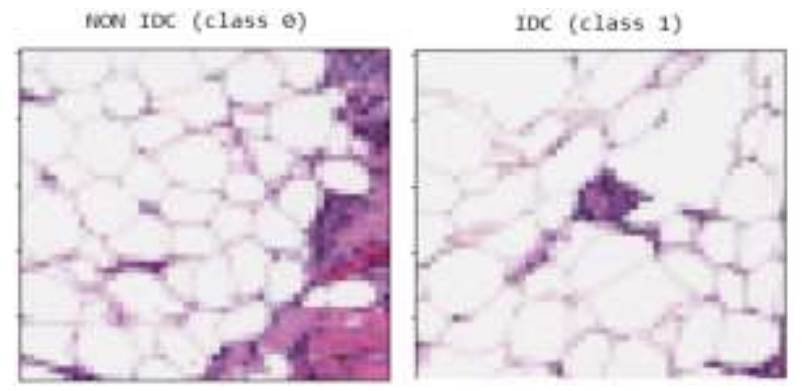

Fig. 13. Classified Classes by GoogLeNet. 


\section{COMPARATIVE ANALYSIS}

As mentioned before, many studies have been performed to predict Breast cancer using machine learning, deep learning, and other techniques based on different imaging data of cancer cells. The following table VIII compares the suggested technique to many other methods used by researchers on various datasets. Despite the fact that previous studies have shown a high degree of accuracy in predicting breast cancer, the proposed method surpassed prediction accuracy.

TABLE VIII. Comparison of Previous Methods AND the Proposed METHOD

\begin{tabular}{|l|l|l|l|}
\hline Studies & Datasets & Models & Accuracy \\
\hline \multirow{2}{*}{\begin{tabular}{l}
\multirow{2}{*}{$\begin{array}{l}\text { Proposed } \\
\text { study }\end{array}$} \\
\cline { 3 - 4 }
\end{tabular}} & \multirow{2}{*}{$\begin{array}{l}\text { Breast histopathology images } \\
\text { from kaggle }\end{array}$} & AlexNet & $96.74 \%$ \\
\cline { 3 - 4 } & & VGG19 & $94.83 \%$ \\
\cline { 3 - 4 } & & Xception & $90.72 \%$ \\
\cline { 3 - 4 } & $\begin{array}{l}\text { Wisconsin Breast Cancer } \\
\text { (original) datasets }\end{array}$ & GoogLeNet & $\mathbf{9 7 . 8 0 \%}$ \\
\cline { 3 - 4 }$[9]$ & - & SVM-RBF & $96.84 \%$ \\
\hline$[11]$ & Breast cancer datasets & Decision tree & $69.23 \%$ \\
\hline$[12]$ & $\begin{array}{l}\text { Wisconsin Breast Cancer } \\
\text { (original) datasets }\end{array}$ & $\begin{array}{l}\text { Neuron fuzzy } \\
\text { methods }\end{array}$ & $95.06 \%$ \\
\hline$[14]$ & $\begin{array}{l}\text { Wisconsin Breast Cancer } \\
\text { (original) datasets }\end{array}$ & SVM & $97.13 \%$ \\
\hline$[24]$ & Image data from Mayo Clinic & SD-CNN & $90 \%$ \\
\hline$[25]$ & Kaggle 162 H\&E & CNN & $87 \%$ \\
\hline$[26]$ & & & \\
\hline
\end{tabular}

\section{CONCLUSION}

Breast cancer is a deadly disease that has claimed the lives of many people in both emerging and industrialized countries around the world. Breast cancer is the second leading cause of cancer death among females in industrialized countries and the first among females in developing countries. According to recent estimates, one of every eight people in Bangladesh will grow breast cancer over their lifetime. As a result, the fight against cancer is far from over. The main focus of this paper is to detect breast cancer that an early stage using the images of IDC cells in the breast. To complete the work, CNN models are used to classify the image data. AlexNet, InceptionV3, VGG19, Xception and GoogLeNet are the algorithms used in the classification process. The algorithms separately are successful in predicting the disease. However, they vary in accuracy of the prediction. It is seen that GoogLeNet has a much higher accuracy rate of $97.80 \%$ compared to the AlexNet and InceptionV3 with $96.74 \%$ and $92.48 \%$. And VGG19 and Xception with $94.83 \%$ and $90.72 \%$. GoogLeNet has higher Precision, Recall, and F1 scores than the other two models. However the models are only tested for the data images available in the Breast histopathology images dataset. The models will further be studied on more dataset to ensure that they can be universally used to detect breast cancer. Using the same process, it is also to be studied that other diseases such as liver cancer, colon cancer, ovarian cancer, etc., can be diagnosed efficiently and with high accuracy at a very early stage.

\section{REFERENCES}

[1] Sharma, G. N., Dave, R., Sanadya, J., Sharma, P., \& Sharma, K. K. (2010). Various types and management of breast cancer: an overview. Journal of advanced pharmaceutical technology \& research, 1(2), 109126.

[2] P. Ghosh et al., "Efficient Prediction of Cardiovascular Disease Using Machine Learning Algorithms With Relief and LASSO Feature Selection Techniques," in IEEE Access, vol. 9, pp. 19304-19326, 2021, doi: 10.1109/ACCESS.2021.3053759.

[3] M. A. Islam, S. Akter, M. S. Hossen, S. A. Keya, S. A. Tisha and S. Hossain, "Risk Factor Prediction of Chronic Kidney Disease based on Machine Learning Algorithms," 2020 3rd International Conference on Intelligent Sustainable Systems (ICISS), 2020, pp. 952-957, doi: 10.1109/ICISS49785.2020.9315878.

[4] F.M. Javed Mehedi Shamrat, Md. Asaduzzaman, A.K.M. Sazzadur Rahman, Raja Tariqul Hasan Tusher, Zarrin Tasnim "A Comparative Analysis of Parkinson Disease Prediction Using Machine Learning Approaches" International Journal of Scientific \& Technology Research, Volume 8, Issue 11, November 2019, ISSN: 2277-8616, pp: 2576-2580. [Asif].

[5] Cireşan D C, Giusti A, Gambardella L M, and Schmidhuber J (2013) Mitosis Detection in Breast Cancer Histology Images with Deep Neural Networks, pp 411-418 (Springer Berlin Heidelberg, Berlin, Heidelberg). doi:10.1007/978-3-642-40763-5_51. Available: https://link.springer.com/chapter/10.1007/978-3-642-40763-5_51.

[6] Xu Y, Jia Z, Wang L-B, Ai Y, Zhang F, Lai M, Eric I, and Chang C (2017) Large scale tissue histopathology image classification, segmentation, and visualization via deep convolutional activation features. BMC Bioinform. 18: 281. Available: https://bmcbioinformatics.biomedcentral.com/articles/10.1186/s12859017-1685-x.

[7] Gao M, Bagci U, Lu L, Wu A, Buty M, Shin H-C, Roth H, Papadakis G Z, Depeursinge A, Summers R M, Xu Z, and Mollura D J (2018) Holistic classification of ct attenuation patterns for interstitial lung diseases via deep convolutional neural networks. Comput. Methods Biomech. Biomed. Eng. Imaging Vis. 6: 1-6. doi:10.1080/21681163.2015.1124249. Available: https://pubmed.ncbi .nlm.nih.gov/29623248/.

[8] Xie W, Noble J A, and Zisserman A. Microscopy cell counting and detection with fully convolutional regression networks. Computer Methods in Biomechanics and Biomedical Engineering: Imaging \& Visualization, 2016, pp. 1-10. Available: https://www.tandfonline. com/doi/abs/10.1080/21681163.2016.1149104.

[9] Chaurasia, Vikas and Pal, Saurabh, Data Mining Techniques: To Predict and Resolve Breast Cancer Survivability (June 29, 2017). International Journal of Computer Science and Mobile Computing IJCSMC, Vol. 3, Issue. 1, January 2014, pg.10 - 22, Available at SSRN: https://ssrn.com/abstract=2994925.

[10] Djebbari A, Liu Z, Phan S, Famili F. An ensemble machine learning approach to predict survival in breast cancer. International Journal of Computational Biology and Drug Design. 2008 ;1(3):275-294. DOI: 10.1504/ijcbdd.2008.021422. Available: https://europepmc.org/article/ $\mathrm{med} / 20054993$.

[11] S. Aruna and L. V Nandakishore, "KNOWLEDGE BASED A NALYSIS OF VARIOUS STATISTICAL TOOLS IN DETECTING BREAST CANCER", Computer Science \& Information Technology (CS \& IT), pp. 37-45, 2011.

[12] Christobel, A. "An Empirical Comparison of Data Mining Classification Methods," vol. 3, no. 2, pp. 24-28, 2011. Available: https://www.semanticscholar.org/paper/An-Empirical-Comparison-ofData-Mining-MethodsChristobel/ba873f0723d244c6f47c58d931747108cf26abc9.

[13] A. Pradesh, "Analysis of Feature Selection with Classification: Breast Cancer Datasets," Indian J. Comput. Sci. Eng., vol. 2, no. 5, pp. 756763, 2011.

[14] Thorsten J. Transductive Inference for Text Classification Using Support Vector Machines. Icml. 1999;99:200-209. doi:10.4218/etrij.10.0109.0425. 
[15] L. Ya-qin, W. Cheng, and Z. Lu, "Decision tree based predictive models for breast cancer survivability on imbalanced data," pp. 1-4, 2009. Available: https://www.infona.pl/resource/bwmeta1.element.ieee-art000005162571/tab/summary.

[16] D. Delen, G. Walker, and A. Kadam, "Predicting breast cancer survivability: a comparison of three data mining methods," Artif. Intell. Med., vol. 34, pp. 113-127, $2005 . \quad$ Available: https://www.sciencedirect.com/science/article/pii/S0933365704001010.

[17] Hosny, K. M., Kassem, M. A., \& Fouad, M. M. (2020). Classification of skin lesions into seven classes using transfer learning with AlexNet. Journal of digital imaging, 33(5), 1325-1334.

[18] Zarrin Tasnim, Sovon Chakraborty, F. M. Javed Mehedi Shamrat, Ali Newaz Chowdhury, Humaira Alam Nuha, Asif Karim, Sabrina Binte Zahir and Md. Masum Billah, "Deep Learning Predictive Model for Colon Cancer Patient using CNN-based Classification" International Journal of Advanced Computer Science and Applications(IJACSA), 12(8), 2021. http://dx.doi.org/10.14569/IJACSA.2021.0120880.

[19] Dong, N., Zhao, L., Wu, C. H., \& Chang, J. F. (2020). Inception v3 based cervical cell classification combined with artificially extracted features. Applied Soft Computing, 93, 106311.

[20] Olah, C., Cammarata, N., Schubert, L., Goh, G., Petrov, M., \& Carter, S. (2020). An overview of early vision in inceptionv1. Distill, 5(4), e00024-002.

[21] Hirano, G., Nemoto, M., Kimura, Y., Kiyohara, Y., Koga, H., Yamazaki, N., ... \& Nagaoka, T. (2020). Automatic diagnosis of melanoma using hyperspectral data and GoogLeNet. Skin Research and Technology, 26(6), 891-897.

[22] F. M. Javed Mehedi Shamrat, P. Ghosh, M. H. Sadek, M. A. Kazi and S. Shultana, "Implementation of Machine Learning Algorithms to Detect the Prognosis Rate of Kidney Disease," 2020 IEEE International Conference for Innovation in Technology (INOCON), Bangluru, India, 2020, pp. 1-7, doi: 10.1109/INOCON50539.2020.9298026.

[23] P. Ghosh, S. Azam, K. M. Hasib, A. Karim, M. Jonkman, A. Anwar, "A Performance Based Study on Deep Learning Algorithms in the Effective Prediction of Breast Cancer," International Joint Conference on Neural Networks (IJCNN 2021), 2021.

[24] Hiba Asri, Hajar Mousannif, Hassan Al Moatassime, Thomas Noel, "Using Machine Learning Algorithms for Breast Cancer Risk Prediction and Diagnosis", Procedia Computer Science, Volume 83, 2016, Pages 1064-1069, ISSN 1877-0509, https://doi.org/10.1016/j.procs.2016.04.224.

[25] Gao, F., Wu, T., Li, J., Zheng, B., Ruan, L., Shang, D., \& Patel, B. (2018). SD-CNN: A shallow-deep CNN for improved breast cancer diagnosis. Computerized Medical Imaging and Graphics, 70, 53-62.

[26] Alanazi, S. A., Kamruzzaman, M. M., Islam Sarker, M. N., Alruwaili, M., Alhwaiti, Y., Alshammari, N., \& Siddiqi, M. H. (2021). Boosting breast cancer detection using convolutional neural network. Journal of Healthcare Engineering, 2021. 